\title{
Mit agilen Blended Learning-Methoden erfolgreich durch die Pandemie: Was Unternehmen von den Hochschulen lernen können
}

\author{
Charlotte Kätzel · Carolin Durst
}

Eingegangen: 25. Januar 2021 / Angenommen: 12. März 2021 / Online publiziert: 9. April 2021

(C) Der/die Autor(en) 2021

Zusammenfassung Die Corona-Krise hat immer noch massive Auswirkungen auf das Gros der gesellschaftlichen Bereiche - auch auf den der schulischen und universitären Bildung. In diesem Zusammenhang gab es bereits vielfältige Diskussionen in deren Rahmen dem deutschen Bildungssektor häufig angekreidet wurde, er sei nicht in der Lage, einer Pandemie dieses Ausmaßes derzeit eine adäquate Reaktion entgegenzusetzen, die nicht nur den Schutz der Lernenden, sondern auch deren schulisches bzw. universitäres Vorankommen sicherstellt.

Dabei mangelt es den Bildungseinrichtungen angesichts der Umsetzung einer funktionierenden digitalen Lehre oft nicht nur an einer geeigneten IT-Infrastruktur, sondern auch an entsprechend ausgebildeten Dozenten sowie den richtigen didaktischen Methoden und Tools.

Dass digitales Lehren, Lernen und Zusammenarbeiten aber auch funktionieren kann, zeigt dieser Beitrag anhand des Beispiels des neuen Master-Studiengangs Digital Marketing an der Hochschule Ansbach für angewandte Wissenschaften. Mit der Hilfe von agilen Blended Learning Methoden konnte der Studiengang nicht nur erfolgreich trotz der Erschwernisse des Corona-Wintersemesters 2020/21 eingeführt werden, sondern auch eine sichere und effektive Lehr-Lern-Situation für die Studierenden und Lehrenden etabliert werden. Digitale Vorträge, Workshops und Präsentationen sind auch für Unternehmen zum New Normal geworden. Wie Unternehmen von den die angewandten Strategien und eingesetzten Tools der Hochschule Ansbach profitieren können, zeigt dieser Beitrag.

\footnotetext{
C. Kätzel $(\square)$

Servicecenter für digitale Lehre und Didaktik, Hochschule Ansbach,

Residenzstraße 8, 91522 Ansbach, Deutschland

E-Mail: charlotte.kaetzel@hs-ansbach.de

C. Durst

Fakultät Wirtschaft, Hochschule Ansbach, Residenzstraße 8, 91522 Ansbach, Deutschland
} 
Schlüsselwörter Blended Learning · Agilität · Digital Marketing · Digitalisierung

\title{
Succeeding During the Pandemic with Agile Blended Learning Methods: What Companies can Learn from Universities
}

\begin{abstract}
The Corona crisis still has massive effects on most areas of society-including school and university education. In this context, there have already been various discussions in which the German education sector has often been criticized for not being able to counter a pandemic of this magnitude with an adequate response that not only protects the learners, but also ensures their educational or academical progress. In view of the implementation of functioning digital teaching, educational institutions often lack not only a suitable IT infrastructure, but also appropriately trained teachers and the right didactic methods and tools. This article shows how digital teaching, learning and collaboration can be successful, using the example of the new Master's program Digital Marketing at Ansbach University of Applied Sciences. With the help of agile Blended Learning methods, the course could not only be successfully introduced despite the difficulties of the Corona winter semester of 2020/21, but also a safe and effective teaching-learning situation was established for the students and teachers. Digital lectures, workshops and presentations have also become the new normal for companies. This article shows how companies can benefit from the strategies and tools used by Ansbach University of Applied Sciences.
\end{abstract}

Keywords Blended Learning $\cdot$ Agility $\cdot$ Digital Marketing $\cdot$ Digitalization

\section{Digitalisierung als Faktor zur Aufrechterhaltung der Wettbewerbsfähigkeit während der Krise}

Eine funktionierende digitale Infrastruktur sowie die grundlegende digitale Bildung nicht nur von Schulkindern und Studierenden, sondern auch der breiten Bevölkerung ist für ein Land von entscheidender Bedeutung in Hinblick auf das Bewältigen einer globalen Krise. Dies hat die fortdauernde Ausnahmesituation, wie sie die Corona-Pandemie mit sich gebracht hat, seit Beginn des Jahres 2020 gezeigt. So wurde es plötzlich in nahezu allen gesellschaftlichen Bereichen notwendig, dort, wo noch nicht geschehen, alltägliche Abläufe in digitale Prozesse zu überführen, Arbeit nach Möglichkeit ins Home-Office zu verlagern und die Bereitstellung entsprechender IT-Infrastrukturen sicherzustellen. In vielen Bereichen mussten diesbezüglich in kurzer Zeit wahre Kraftakte gestemmt werden, so dass die Pandemie geradezu katalysatorische Wirkung in Hinblick auf digitale Transformationen entfaltet hat. Es gibt Stimmen, wie etwa Professor Philip Meissner vom European Center for Digital Competitiveness der ESCP Business School Berlin, die gar von einer digitalen Revolution sprechen, die durch die Pandemie in Gang gebracht wurde. Doch mit dieser Revolution kann nicht überall in gleichem Maße Schritt gehalten werden. Im direkten Ländervergleich zeigten sich in dieser Hinsicht schnell große Unterschiede im Zuge der Bewältigung der Krise - mit vermutlich weitreichenden Folgen: 
„Die Art und Weise, wie die Regierungen diesen Übergang gestalten, wird maßgeblich darüber entscheiden, wie wettbewerbsfähig und wohlhabend ihre Länder in den kommenden Jahrzehnten sein werden.“, so Meissner (European Center for Digital Competitiveness 2020). Diese Feststellung kann ebenso auf die gesellschaftlichen Bereiche innerhalb eines Landes übertragen werden. So haben etwa deutsche Unternehmen seit Beginn der Krise mit drastischen wirtschaftlichen Auswirkungen zu kämpfen. Es hat sich dabei gezeigt, dass diejenigen Unternehmen, die bereits vor der Pandemie digitalisierte Geschäftsmodelle aufweisen konnten, besser durch die Krise kommen (it-daily.net 2020). Unternehmen, die zuvor nicht oder nur wenig in die Digitalisierung investiert hatten, haben im Umkehrschluss häufig gravierende Probleme damit, während der Krise (und voraussichtlich darüber hinaus) wettbewerbsfähig zu bleiben.

In einer ähnlichen Situation befindet sich auch die deutsche Bildungslandschaft, der ein gravierender Mangel in der digitalen Bildungsinfrastruktur und ein deutliches Zurückfallen im europäischen Vergleich attestiert wird - den Digitalisierungsgrad betreffend findet sich Deutschland gar als europäisches Schlusslicht wieder (Wiesinger et al. 2020).

Doch auch in diesem Bereich finden sich Beispiele für eine gelungene Bewältigung der Krise, die durch bereits bestehende digitalisierte Konzepte in Kombination mit angepassten Methoden den Widrigkeiten die Stirn bieten konnten und können. Ein Blick auf den innovativen, neuen Masterstudiengang Digital Marketing der Hochschule Ansbach für angewandte Wissenschaften am Campus Rothenburg soll deutlich machen, welcher Voraussetzungen es bedarf, um in der derzeitigen Ausnahmesituation funktionierende Lehr-/Lernsituationen sowie kollaboratives Arbeiten zu ermöglichen - dabei können auch Unternehmen von den Learnings profitieren, die durch die Erprobung verschiedener Tools, Methoden und Strategien im Studiengang gewonnen wurden und die ein schnelles und effektives „Switchen“ zwischen traditionellem und digitalem Betrieb ermöglichen.

\section{Das Konzept des Blended Learning-Studiengangs Digital Marketing}

Der Studiengang Digital Marketing startete mitten im Pandemiejahr 2020 zum Wintersemester 2020/21. Bei der Konzeption des Masterstudiengangs im Vorfeld hatte der Fokus dabei nicht etwa darauf gelegen, ein „krisensicheres“ Studium zu ermöglichen; vielmehr war es Ziel, Full Stack Marketer auszubilden und strategisches sowie operatives digitales Marketingwissen im Rahmen eines möglichst adäquaten Formats, des Blended Learning-Formats, zu vermitteln. „Blended Learning (engl. Blender $=$ Mixer) ist ein internet- bzw. intranetgestütztes Lernsystem, das problemorientierte Workshops mit meist mehrwöchigen Phasen des selbstgesteuerten Lernens auf der Basis von Web Based Trainings und der Kommunikation über ein Learning Management-System bedarfsgerecht miteinander verknüpft." (Erpenbeck et al. 2015) Es handelt sich also um ein Konzept, das die Vorteile von Präsenz- und Onlinelehre aufgreift und verknüpft. Dabei ist auf der Seite des digitalen Selbststudiums vor allem die hohe Flexibilität zu nennen, die Studierende bzw. allgemein Lernende durch sie gewinnen. Die Lernenden können sich Wissensinhalte in zeitli- 
cher und räumlicher Unabhängigkeit aneignen. Auf diese Weise wird das Studium auch für berufstätige oder (allein-)erziehende Personen geöffnet. Ein Umzug an den Studienort ist nicht mehr unbedingt notwendig. Zudem fördert die Auseinandersetzung mit verschiedensten digitalen (Lern-)Plattformen, Softwares und Tools die Entwicklung von digitalen und sog. future skills, wie etwa Digital Literacy, Digital Learning, Digitale Interaktion und Kollaboration (Kirchherr et al. 2019), die schon jetzt beim Eintritt in das Berufsleben relevant sind und in Zukunft für junge Professionals immer unabdingbarer sein werden. Gerade im Feld des digitalen Marketings sichert das durch das Blended Learning-Format erworbene und geübte Können im Umgang mit digitalen Medien einen klaren Vorteil gegenüber traditionell in Präsenz ausgebildeten Kräften.

Währenddessen birgt der Präsenzanteil des Studiums aber andere, in Selbstlernphasen nur schwer abzubildende Vorteile, wie etwa soziale Aspekte, die keinesfalls unterschätzt werden sollten. So stellt Michael Kerres (2018) fest, dass „,(d)ie Motivation und Bindung der Teilnehmenden steigt, wenn sie nicht mehr nur alleine mit einem Computer interagieren, sondern auch und zugleich eingebunden sind in eine soziale Gruppe und von einer Lehrperson betreut werden. Die Abbruchquote, die bei einem reinen Online-Angebot teilweise recht hoch ist, kann auf diese Weise reduziert werden."

Auch wenn das Konzept Blended Learning sich aus diesen Gründen in den letzten Jahren immer größerer Beliebtheit und Verbreitung erfreut, verspricht die alleinige Umstellung eines Lehr-/Lernangebotes auf Blended Learning noch keinen Erfolg. Denn das Konzept selbst „(...) sagt per se nichts über den Lernerfolg oder die Qualität der Lehre aus, verrät auch nichts zu den zu verwendenden Methoden und Medien, klärt nicht die Intensität und Dauer von Online-Lernphasen oder die Rolle von Präsenzveranstaltungen." (Gerner et al. 2019).

Essentiell für gelungene Blended Learning-Konzepte ist daher, dass die mediengestützten Selbstlernelemente und klassischen Präsenzelemente sinnvoll miteinander verzahnt werden. Bei der Konzeption des Studiengangs war dabei auch der ebenfalls im Blended Learning-Format gestaltete Bachelorstudiengang Interkulturelles Management von großer Bedeutung. Dieser startete erstmals zum Sommersemester 2016 am Campus Rothenburg, dem jüngsten Außenstandort der Hochschule Ansbach, an dem ebenfalls Digital Marketing angesiedelt ist. Das wertvolle Wissen, das aus den Erfahrungen sowohl von Lehrenden- als auch aus Studierendenseite gewonnen werden konnte, floss in die Gestaltung des Masterprogramms Digital Marketing ein. Als wichtige Komponente des Studiengangs Digital Marketing kam etwa wie schon beim Studiengang Interkulturelles Management die Methode des Flipped bzw. Inverted Classroom zum Tragen, die sich in vielen Blended Learning-Szenarien gewinnbringend einsetzen lässt. Beim Flipped Classroom eignen sich Studierende Wissensinhalte im Rahmen von Selbstlernphasen an, z. B. mit Hilfe von Lernvideos oder anderen bereitgestellten Materialien. Die anschließenden Präsenzveranstaltungen werden dann für soziale Prozesse wie Anwendung, Vertiefung oder Diskussion verwendet (Gerner et al. 2019).

Während die Lehrenden im Masterprogramm Digital Marketing zumeist schon vor Beginn der Corona-Pandemie Flipped Classroom-Ansätze verfolgt hatten, musste hier nicht mehr allzu viel Zeit in das Heraussuchen passender Tools, die Überarbei- 
tung bereits bestehender Unterrichtsmethoden sowie -materialien gesteckt werden. Tatsächlich bedeutete für viele bis zu Beginn der Pandemie in klassischer Präsenzform Lehrende die Umstellung von Unterrichtsmethoden und damit auch -materialien sowie die damit verknüpfte veränderten Arbeitsweise eine zuweilen hohe Hürde und einen nicht zu vernachlässigenden Mehraufwand. Es ist jedoch erwähnenswert, dass dieser Mehraufwand sich nach einigen Semestern der digitalen bzw. hybriden Lehre durchaus gut amortisieren kann. So kann es etwa einen hohen Aufwand mit sich bringen, einen Foliensatz mit Hilfe eines Autorentools wie bspw. Adobe Captivate (in dessen Anwendung man sich zunächst auch noch schulen lassen muss) in ein interaktives Lernprogramm umzusetzen. Das fertige Lernprogramm kann danach jedoch in folgenden Semestern gut wiederverwertet und ggf. angepasst werden.

Da richtig aufgezogenes Blended Learning die Vorteile von Präsenz- und Fernlehre gewinnbringend in sich vereint, lohnt es sich, die Corona-Pandemie in dieser Hinsicht als Chance zu begreifen: Vor der Ausnahmesituation schreckten viele Lehrende noch vor dem Einsatz digitaler oder hybrider Lehrformate zurück - aus Gründen wie etwa der Unerfahrenheit mit digitaler Technologie, Sorgen wegen wachsenden Aufwands und hinsichtlich urheber- und datenschutzrechtlicher Problematiken. Inzwischen konnten sich die meisten dieser Lehrenden, wenn auch ,gezwungenermaßen“, einen guten Einblick über die Möglichkeiten digitaler Lehre verschaffen und für sich und die eigene Lehre Pros und Contras in diesem Zusammenhang ermitteln, Ängste und Sorgen konnten zumindest zum Teil entkräftet werden. Die Herausforderung für die Zeit nach Corona wird es daher sein, Lehrende von der Beibehaltung sinnvoller digitaler Lehrelementen zu überzeugen und sie bei der effektiven Verankerung in der eigenen Lehre bzw. bei der Verzahnung mit klassischen Formaten zu gelungener hybrider Lehre zu bestärken. Hierfür müssen die Lehrenden von entsprechenden Einrichtungen an der heimischen Hochschule didaktisch und technisch unterstützt werden, aber auch die Schaffung geeigneter rechtlicher, it-infrastruktureller und finanzieller Rahmenbedingungen wird dafür essenziell sein. Im Rahmen virtueller Onboardings, wie sie auch im Rahmen des Studiengangs Digital Marketing bereits durchgeführt werden, können außerdem neue Lehrende direkt mit dem Blended Learning-Prinzip und allen notwendigen Tools vertraut gemacht werden.

\section{Agiles Blended Learning in der Krise - Von der Präsenz zur Online- Präsenz und umgekehrt}

Nachdem sich das Blended Learning-Prinzip in der Vergangenheit bewährt und den Studierenden bereits große zeitlich-räumliche Flexibilität geboten hat, mussten im Laufe der Corona-Pandemie trotzdem weiterhin Anpassungen getroffen werden. Obwohl der Studiengang bereits so ausgelegt war, dass die Studierenden vergleichsweise wenig Präsenzzeit am Campus absolvieren mussten, galt es, auch diese Präsenzen - zur Sicherheit der Studierenden und Lehrenden - weiter einzuschränken bzw. schließlich gänzlich aufzuheben. Es musste Agilität bewiesen werden, also die Fähigkeit, auf sich verändernde Rahmenbedingungen schnell und kreativ reagieren zu können (Neun 2020). Diese neuen Rahmenbedingungen brachten zudem 
einen besonders hohen Grad an Unvorhersehbarkeit mit sich; sowohl Lehrende als auch Lernende wussten oft nicht, ob und inwiefern Lehr-Lernsituationen sich in absehbarer Zukunft verändern würden. Diesen Unsicherheiten wurde aber nicht mit Ausweichen oder Abwarten entgegengetreten, stattdessen konnte ihnen im Sinne agiler Ansätze mit einem aktiven, gegenseitig unterstützenden Lernprozess aller Beteiligten entgegengewirkt werden. Dieser bedeutet für die Lehrenden nicht nur, auf schnelles Switchen zwischen Fern- und Präsenzlehre vorbereitet zu sein, sondern auch auf lebenslanges Lernen auf Augenhöhe mit den Studierenden. So galt es etwa, für Reverse Coaching durch die Studierenden, die meist Digital Natives sind, offen zu sein - ein Kernelement von Agilität (Baltes und Freyth 2017). Es kam beispielsweise zu Situationen, in denen Studierende die Lehrenden beim Einsatz bestimmter digitaler Tools unterstützten und es entwickelten sich in der gegenseitigen Unterstützung oft fruchtbare Austauschsituationen. Der Austausch war generell ein entscheidendes Element im agilen Bewältigen der Krise: An der Hochschule wurde ein regelmäßiger Erfahrungsaustausch zur digitalen Lehre ins Leben gerufen, in dessen Rahmen Lehrende Best Practices vorstellten, aber auch Unsicherheiten und Problemstellungen diskutierten (Abb. 1). Dabei wurde ein iterativer Lernprozess für alle Beteiligten angestoßen, der für die einzelnen Lehrpersonen ein stetiges Nachbessern in der Kombination Ihrer eingesetzten technischen bzw. digitalen Tools und didaktischen Methoden ermöglichte.

Das Blended Learning-Konzept konnte sich als agil und damit „krisenfest“ bewähren: Ohne großen Aufwand wurde von einem Tag auf den anderen auf reinen Online-Betrieb geswitcht. Wo noch Unsicherheiten auftraten, wurde durch offenen Austausch, Reverse Coaching und den Anstoß iterativer Lernprozesse die Chance in der Krise ergriffen. Was für klassische Präsenzstudiengänge vielerorts ein großes Problem darstellte, konnte hier in nur kurzer Zeit und mit vergleichsweise geringer Anstrengung bewältigt werden. Im Folgenden werden die relevanten Aspekte vorge-

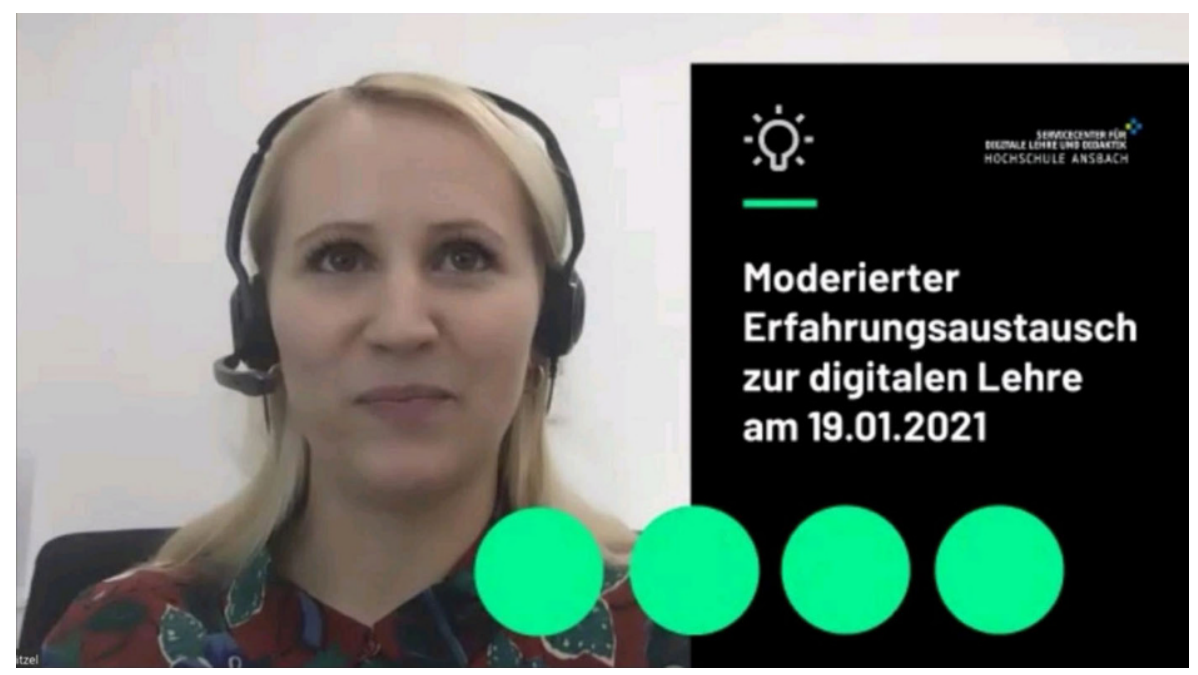

Abb. 1 Erfahrungsaustausch zur digitalen Lehre an der Hochschule Ansbach 
stellt, welche diese Vorgehensweise ermöglichen, und auf den Unternehmenskontext übertragen (Abb. 1).

\subsection{Learning Management System}

Für die ohnehin planmäßig in Digital Marketing angelegten Selbstlernphasen existierte bereits ein Learning Management System (Moodle), auf dem alle Kurse standardmäßig angelegt sind. Auf dieser webbasierten Plattform werden digitale Lerninhalte sowie wichtige Informationen eingestellt. Die Kommunikation zwischen den Lehrenden und Studierenden sowie den Studierenden untereinander erfolgt über Foren oder Mailingsystem. Zudem können sich Studierende in Gruppen eintragen, Evaluationen vornehmen und vieles mehr. Auch E-Prüfungen können mit Moodle umgesetzt werden. Learning Management Systeme wie Moodle sind zudem häufig Freeware und können auf einem eigenen Server von der jeweiligen IT-Abteilung betrieben werden. Alternativ gibt es auch Anbieter, die datenschutzkonformes Hosting sowie Support für die Systeme anbieten. An der Hochschule Ansbach wird Moodle extern gehostet, inhouse gibt es jedoch mit dem Servicecenter für digitale Lehre und Didaktik eine Einrichtung, deren Mitarbeiter das System administrieren und Support für Lehrende und Studierende sowie regelmäßige Trainings anbieten. Während gerade im schulischen Bereich in vielen Bundesländern, allen voran BadenWürttemberg und Nordrhein-Westfalen, über weitreichende Ausfälle von Moodle geklagt wurde, gab es an der Hochschule Ansbach daher auch keine nennenswerten Probleme mit Störungen von Moodle, die über ein ,normales“ Maß hinausgingen. Selbst bei auf der Plattform stattfindenden digitalen Abschlussprüfungen, die das System mit hoher Belastung konfrontierten, gab es keine Ausfälle. An dieser Stelle zu betonen, dass das Hosting eines Systems wie Moodle nicht auf die leichte Schulter genommen werden sollte, sondern bei der Vergabe an Dienstleister bzw. auch beim Inhouse Hosting darauf geachtet werden sollte, dass im Falle von Phasen hoher Belastung genügend Kapazität - was Server sowie personelle Betreuung betrifft sichergestellt werden kann.

Zur Nutzung von Moodle wird ansonsten nur ein internetfähiges Endgerät auf Seiten der Nutzer benötigt. Da es eine entsprechende App gibt, kann das System auch bequem mobil via Smartphone genutzt werden.

Das Learning Management System spielt für die Agilität eine wichtige Rolle: Moodle wird im Rahmen der Online Lehre von zu Hause oder unterwegs genutzt, aber auch ergänzend im klassischen Präsenzunterricht. Dies kann entweder im PCPool oder auch im Klassenzimmer geschehen - hier etwa via Smartphone oder Notebook, ganz nach der auch im Studiengang Digital Marketing gelebten zeitgemäßen didaktischen Methode Bring Your Own Device.

Von großer Bedeutung war bei der Umstellung auf gänzlich digitale Lehre die bereits bestehende Vertrautheit aller Beteiligten mit dem Learning Management System. Die Lehrenden hatten bereits vorher digitale Lerninhalte erstellt, wie etwa (interaktive) Lernvideos, Podcasts oder Quizze, die neben klassischen Lerninhalten wie Texten auf der Lernplattform zur Verfügung gestellt werden können. An dieser Stelle wurden im Studiengang sehr gute Erfahrungen mit Onboardings für neue Lehrende gemacht, in deren Rahmen diese in der Nutzung aller wichtigen Funktionen 
des Learning Management Systems geschult werden. Auch die Studierenden werden von Anfang an mit der digitalen Lernumgebung vertraut gemacht und finden sich problemlos zurecht.

\subsubsection{Learnings für Unternehmen}

Was für Universitäten und Hochschulen Learning Management Systeme sind, sind für Unternehmen Wissensmanagementsysteme. Diese stellen ein zentrales Instrument für die interne Kommunikation dar (Meier et al. 2015). Wissensmanagementsysteme unterstützen den Prozess der Identifizierung, Sammlung, Speicherung, Bewertung und Weitergabe aller wertvollen Informationen. Für Unternehmen bilden diese Systeme die Grundlage, um gerade in Krisenzeiten, die persönliche Treffen von mehreren Personen ausschließen, die Zusammenarbeit der Mitarbeiter und so die allgemeine Geschäftsfähigkeit sicherzustellen. Unternehmen, die bereits im regulären Betriebsablauf auf Wissensmanagementsysteme wie Microsoft SharePoint oder Atlassian Confluence setzen, können viel schneller und unkomplizierter - sozusagen agil - auf einen digitalen Geschäftsbetrieb umstellen. Wie auch beim gewohnten Einsatz von Moodle im Studiengang Digital Marketing sind dann auch die Mitarbeiter bereits mit dem System vertraut und eine überstürzte Umgewöhnung/Schulung ist nicht mehr nötig.

\subsection{Webkonferenzsysteme und weitere Devices für Online-Präsenzen}

Während in Hinblick auf die Selbstlernphasen im Masterprogramm also vor allem dank des sich bereits im Einsatz befindlichen Learning Management Systems Moodle keine weiteren Anpassungen nötig waren, musste im Bereich der eigentlich als traditionelle Präsenzveranstaltungen geplanten Phasen im Rahmen des Studiums eine Anpassung stattfinden. Die wichtigste Ressource hierfür war ein performantes Webkonferenzsystem. Eine Herausforderung bei der Implementierung eines solchen Systems findet sich mit Blick auf datenschutzrechtliche Aspekte - diese sollten dringend rechtzeitig abgeklärt werden. Es hat sich außerdem gezeigt, dass das Organisieren von Grundlagenschulungen zur Einführung eines Systems auch hier sehr empfehlenswert ist und sich positiv auf die Qualität der Live Online-Präsenzen auswirkt sowie allen Beteiligten deutlich mehr Sicherheit gibt. Wie auch für die Nutzung von Moodle wird für Webkonferenzsysteme wie etwa Zoom nur ein internetfähiges Endgerät benötigt, das aber auch mit integriertem Mikrofon und Webcam ausgestattet sein sollte. Damit eine hohe Qualität der Online-Lehre gesichert werden kann, wird in erster Linie den Dozierenden, aber auch den Studierenden, die Nutzung eines Headsets empfohlen, da die Audioqualität während der Übertragung so deutlich gesteigert werden kann. Untersuchungen zeigen, dass Audioqualität im virtuellen Raum wesentlich relevanter als Bildqualität ist und in hohem Maße zu einer bleibenden Aufmerksamkeit der Teilnehmenden beitragen kann (Gerstbach 2020). Sollte ein Gerät über keine integrierte Webcam verfügen, wird die Anschaffung einer externen Webcam empfohlen - durch die, zumindest zeitweise, Freischaltung der Kamerabilder, wird die gefühlte Distanz im Rahmen von virtuellen Veranstaltungen gemindert und der fehlende soziale Kontakt zumindest teilweise hergestellt. 


\subsubsection{Learnings für Unternehmen}

Für Unternehmen tun sich in Hinblick auf die Nutzung von Webkonferenzsystemen die gleichen Vorteile wie für den Bildungsbereich auf - auch wenn es sich bei den in den virtuellen Raum zu übersetzenden Veranstaltungen nicht um Vorlesungen und Sprechstunden, sondern um Team Meetings und Mitarbeitergespräche handelt. Die Empfehlungen hinsichtlich der nötigen Ausstattung können dabei übernommen werden. Im Rahmen von Studiengängen wie Digital Marketing, die auf einem hohen Niveau digitaler Kompetenz stattfinden, werden auch zusätzliche Devices wie Digitizer Boards oder Dokumentenkameras genutzt, die z. B. Live-Visualisierungen während der Online-Präsenzen ermöglichen. Auch im betrieblichen Bereich kann sich der Einsatz solcher Hardware lohnen, um beispielsweise Pitches auf ansprechende Weise online umsetzen zu können. Damit Mitarbeiter den Umgang mit den Devices lernen, sollte auch während des regulären Betriebes entsprechend auf diese gesetzt werden. Wer im Büro bereits mit Smartboards statt Flipcharts gearbeitet hat, kann nicht nur erstellte Präsentationen bequem via USB-Stick auf mobile Devices übertragen, sondern ist auch mit deren Funktionsweise und Bedienung vertrauter.

Für Lehrende und Studierende, die nicht über die nötige technische Ausstattung verfügen bzw. durch kurzfristigen Ausfall Ersatz benötigen, hält der Studiengang Digital Marketing ein Kontingent an verschiedenen Leihgeräten sowie Adaptern aller Art bereit - auch im Unternehmen sollten Leihgeräte für Mitarbeiter entsprechend zur Verfügung stehen, damit der Betrieb im Ernstfall schnell auf digital umgestellt werden kann.

Bei der Nutzung von Webkonferenzsystemen ist eine stabile und leistungsfähige Internetverbindung von hoher Relevanz. Für Mitarbeiter, die im Home-Office über keine oder nur eine schlechte Verbindung verfügen, können nach Möglichkeit im Büro Arbeitsplätze eingerichtet werden, auf die im Notfall zugegriffen werden kann.

\subsection{Wissensvermittlung und Didaktik}

Mit Hilfe des Webkonferenzsystems können ansonsten in Präsenz stattfindende Veranstaltungen und Meetings sehr gut auf die virtuelle Ebene verlegt werden. Je nach Art der Veranstaltung müssen aber in Hinsicht auf die Art der Wissensvermittlung bzw. der didaktischen Aufbereitung Anpassungen im Vergleich zur traditionellen Variante getroffen werden. So können Sprechstunden, kurze Absprachen o. ä. inhaltlich wie gewohnt umgesetzt werden. Anpassungen bedarf es bei längeren Terminen, wie bei klassischen Lehrveranstaltungen - die (Online-) Präsenz muss hier oft grundlegend verändert werden (Kerres 2018). So sollten Ganztagveranstaltungen vermieden werden und eher auf kürzere Sessions gesetzt werden, sowie auch mehr Pausen eingeplant werden: Im Hochschulbereich bietet der auch in Digital Marketing zur Anwendung kommende Flipped Classroom-Ansatz hierfür beste Voraussetzungen, da die Präsenzzeiten gezielter genutzt werden können, um Wissensinhalte zu vertiefen, die die Studierenden sich in den Selbstlernphasen angeeignet haben - etwa mit der Arbeit an verschiedenen Beispielen. Außerdem sollte hier der Raum für Fragen sein. Für längere Input-Phasen sollten die Online Präsenzen hingegen nicht genutzt werden, da während derer die Aufmerksamkeit in diesem Setting ohnehin nur schwer 
dauerhaft gehalten werden könnte. Erfolgt Input, so werden Phasen von Informationsvermittlung (Lehrvortrag etc.) und Informationsverarbeitung (z. B. Gruppenarbeit, Aufgabenbearbeitung) kürzer gestaltet und finden in häufigerem Wechsel statt, als dies in traditionellen Präsenzveranstaltungen der Fall wäre.

Wenn digitale Learning Tools (siehe Abschn. 3.4) eingesetzt werden, sollten diese didaktisch sinnvoll in die Lehrveranstaltung integriert werden. D.h., es sollte stets zuerst ein Lernziel für die entsprechende Lerneinheit festgelegt werden, außerdem die Sozialform (z. B. Gruppenarbeit) bestimmt werden, um dann in einem weiteren Schritt entsprechende Tools zur Umsetzung auswählen zu können. Eine hilfreiche Unterstützung in dieser Hinsicht können Lehr-/Lernmethoden-Karteien darstellen. Mit dem elektronischen Lehrportal der Bergischen Universität Wuppertal (ELP) beispielweise, können über eine Filterfunktion passende Methoden und digitale Applikationen für ein bestimmtes Vorhaben (Sozialform, Lehrphase, ggf. Lernzieltaxonomie) gefunden werden (https://www.elp.uni-wuppertal.de/?language=de).

\subsubsection{Learnings für Unternehmen}

Auch außerhalb des Bildungsbereich sollten die oben genannten Faktoren bei Live Online-Veranstaltungen bedacht werden. Online stattfindende Ganztagesveranstaltungen sollten daher eher vermieden werden und eher auf häufigere, aber dafür kurze Meetings gesetzt werden (höchstens $90 \mathrm{~min}$ ). Bei längeren E-Meetings sollten Unternehmen nach 40-50 min eine Pause von 10-15 min einplanen. Mehr als vier dieser Blöcke am Stück sind nicht empfehlenswert. Auf diese Weise kann vermieden werden, dass die Aufmerksamkeit der Teilnehmenden nachlässt, sie ermüden und/ oder sich durch Smartphone \& Co ablenken lassen. Es sollte dabei ebenso darauf geachtet werden, dass verschiedene Meeting-Elemente bewusst ausbalanciert und abwechslungsreich eingebracht werden (Harnacke 2020).

Harnacke (2020) unterscheidet dabei sieben Besprechungsinhalte.

Für jeden der sieben Besprechungsinhalte gilt es, diese auf die virtuelle Ebene zu heben und durch aktivierende Methoden zu unterstützen. Die im Folgenden

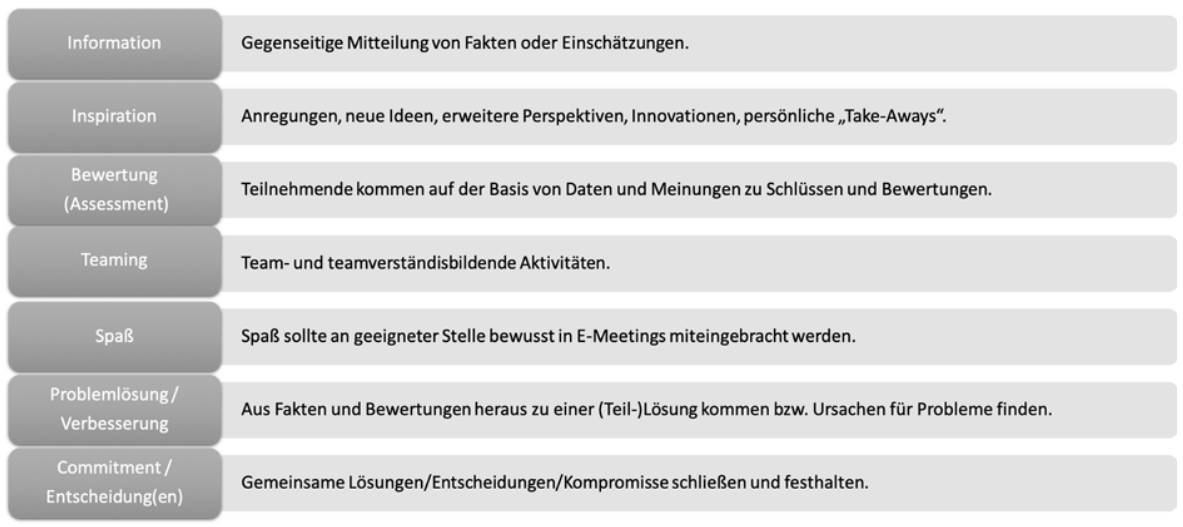

Abb. 2 Die sieben typischen Besprechungsinhalte. (Harnacke 2020) 
dargestellten Erfahrungen aus der Lehre lassen sich 1:1 auf Besprechungen in Unternehmen übertragen.

\subsection{Aktivierende Methoden - Motivation durch richtige Moderation}

Online-Lehre sollte prinzipiell interaktiv und aktivierend gestaltet werden - diese Faustregel kann aber auch auf Veranstaltungen übertragen werden, die nicht im Rahmen von Lehre stattfinden. Das Nutzen aktivierender Methoden ist von großer Bedeutung, damit Teilnehmende in der virtuellen Umgebung, in der sie deutlich schneller ermüden, aufmerksam, aktiv und motiviert bleiben. Beispiele für solche Methoden sind aktivierende, spielerische Ein- und Ausstiegsrunden (am besten mit aktivierter Webcam), das Abfragen von (Vor-) Wissen, Vorerfahrungen oder Meinungen via Abstimmungstools oder Chat, Gamification-Elemente wie Quizze, gemeinsames Brainstorming bzw. Brainwriting oder auch Fragetechniken (Punkteabfrage, Skalierungsabfrage) via digitales Whiteboard (Abb. 3 und 4) sowie sog. Blitzlichter, bei denen alle Teilnehmende etwa über Status-Icons Feedback geben, also ihre Meinung, Zustimmung oder Ablehnung kundtun können. Erfahrungsgemäß kann die Resonanz außerdem signifikant erhöht werden, indem der gewünschte Kanal für Rückmeldungen/Interaktionen an die Teilnehmenden klar kommuniziert wird. So bringt es häufig keinen Erfolg, eine Frage einfach in den Raum zu werfen - stattdessen muss herausgestellt werden, ob Rückmeldungen beispielsweise via StatusIcons, über den Chat oder als Wortmeldung erfolgen sollen.

Zur Aktivierung von Teilnehmenden einer virtuellen Veranstaltung eignet sich auch gut Gruppenarbeit bzw. generell die Aufteilung in Gruppen zu unterschiedlichen Zwecken. So können in Kleingruppen Sachverhalte diskutiert und Aufgaben gelöst werden. In virtuellen Gruppenräumen/Breakout Sessions lernen sich die Teilnehmenden gleichzeitig auch besser kennen und kommunizieren gelöster und

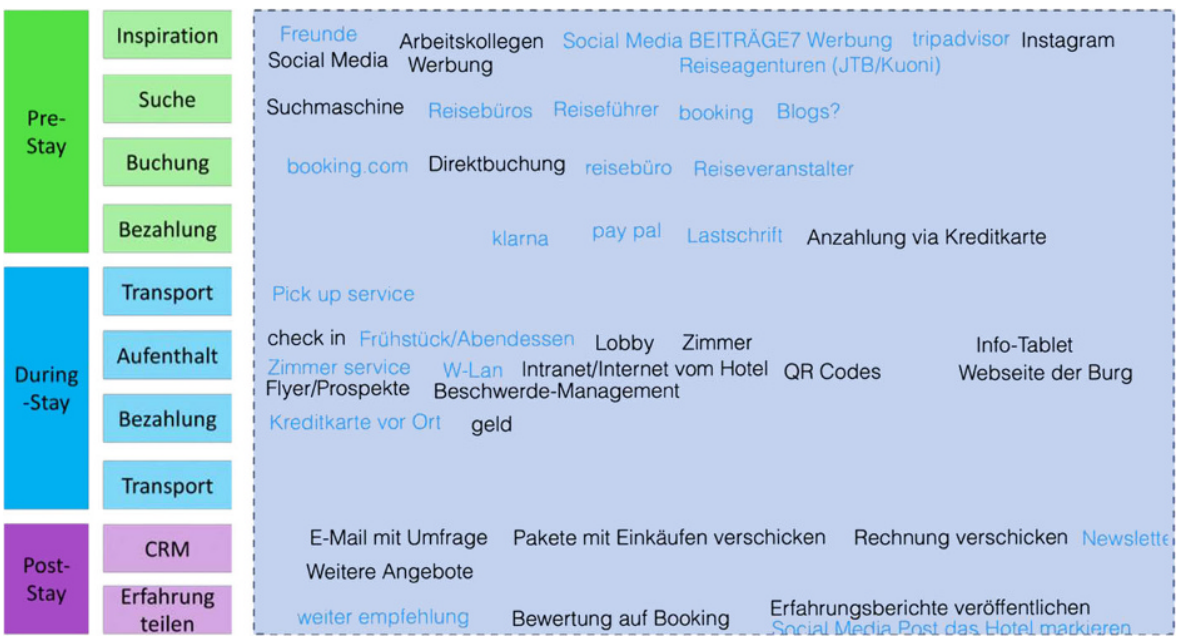

Abb. 3 Whiteboard-Abfrage im Blended Learning Studiengang Interkulturelles Management 
Wie sieht das prozentuale Verhältnis von synchronen

und asynchronen Elementen in Ihrer Lehre aus?

Bitte setzen Sie einen Stempel.

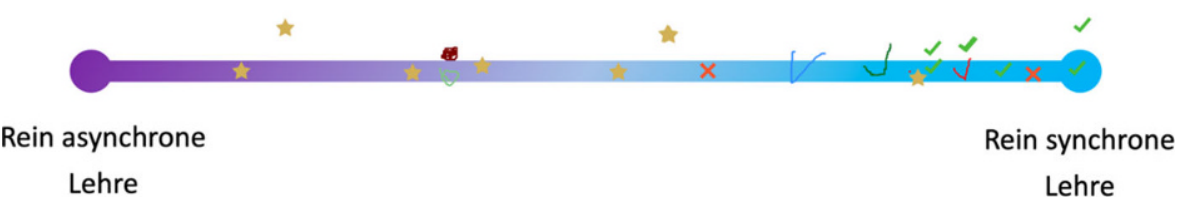

Abb. 4 Whiteboard-Skalierungsabfrage im Rahmen eines virtuellen Erfahrungsaustauschs zur digitalen Lehre

mutiger als im vollständigen Plenum - dieser Effekt kann sich beim Auflösen der Gruppen auf das Verhalten im Plenum übertragen.

Werden Präsentationen gezeigt, so sollte im virtuellen Meeting Raum darauf geachtet werden, dass die Foliengestaltung nach dem Prinzip „Bild vor Wort“ erfolgt einzelne Folien sollten tendenziell weniger Text enthalten und nicht überfrachtet sein. Damit die Aufmerksamkeit der Teilnehmenden aufrechterhalten werden kann, müssen deutlich mehr visuelle Reize gesetzt werden als in klassischen Präsenzveranstaltungen. So sollte der Folienwechsel recht hochfrequent erfolgen, sowie mit Animationen, Unterstreichungen oder Laserpointer gearbeitet werden. Auch das Stichwort Medienwechsel ist für virtuelle Veranstaltungen relevant, da die abwechselnde Einbindung von Videos, Audiofiles, Bildern oder externen Websites ebenfalls zur Aufmerksamkeit der Teilnehmenden beiträgt.

Als Faustregel sollte gelten, etwa alle sieben Minuten ein aktivierendes Element einzubringen (Harnacke 2020). Dies muss nicht immer ein „Tool-Feuerwerk“ bedeuten, sondern kann auch sehr niedrigschwellig erfolgen, wie etwa über eine kurze Feedback-Abfrage mit Emoticons oder die Bitte um eine schnelle Abstimmung mit „Ja“ oder „Nein“ im Chat.

Gerade für fortgeschrittene Lehrende bzw. Moderatoren kann es sich aber auch anbieten, externe Tools einzubinden. Im Rahmen von Digital Marketing werden etwa Audience Response-Systeme wie Pingo oder Kahoot genutzt, die es Teilnehmenden ermöglichen, via Smartphone in Echtzeit auf von den Lehrenden erstellte Fragen zu antworten. Dieses Online Quizzing erhöht nicht nur Zufriedenheit, Engagement, Selbstwirksamkeitserwartung und verbessert Prüfungsleistungen sowie Langzeit-Wissensbehalt (DeSouza und Fleming 2003), sondern hat auch einen aktivierenden Gamification-Effekt. Interessant sind auch Live Q \& As wie frag.jetzt, die die Stellung anonymer Fragen erlauben, die dann etwa am Ende einer Veranstaltung von den Lehrenden beantwortet werden können. Diese Möglichkeit aktiviert häufig auch diejenigen Teilnehmenden, die sich tendenziell aus unterschiedlichen Gründen nicht trauen würden, sich in der Runde zu Wort zu melden. Vor allem im Kontext von Gruppenarbeiten bzw. generell kollaborativem Arbeiten bieten sich auch digitale Pinnwände wie Padlet an oder auch multifunktionale digitale Whiteboards von Anbietern wie Miro, die das (gemeinsame) Anfertigen von Mind Maps, Roadmaps, 
Kanban Boards oder Charts ermöglichen. Viele dieser Tools haben durchaus nicht nur im Bildungsbereich eine effektive Wirkung und können zudem auch außerhalb von Online-Meetings, also im asynchronen Bereich, eingesetzt werden.

\subsection{Softskills}

Es ist wichtig herauszustellen, dass nicht nur technische/digitale Ressourcen für ein funktionierendes Switchen in den Digitalbetrieb (und zurück) nötig sind. Auch solche in Form von entsprechenden Soft Skills der Lehrenden und Studierenden waren für das erfolgreiche Vorgehen von Digital Marketing ausschlaggebend. So erfordern längere Selbstlernphasen zur Wissensaneignung etwa generell ein etwas höheres Maß an Selbstdisziplin, wie auch eine Studie des Forschungs- und Innovationslabors digitale Lehre nach dem ersten digitalen Semester im Sommer 2020 bestätigte (Kreulich et al. 2020). Bei Studierenden, die bereits vor dem Eintreten der Pandemie im Blended Learning-Format studiert haben, ist diese Kompetenz bereits ausgebildet, sodass bei der Umstellung auf komplett digitale Lehre hier keine mit großen Schwierigkeiten verbundene Umgewöhnung erforderlich war.

\subsubsection{Learnings für Unternehmen}

Für Unternehmen ist es mit Blick auf die Ausformung entsprechender Soft Skills der Mitarbeiter empfehlenswert, vermehrt nach New Work Ansätzen zu arbeiten - also etwa Arbeit flexibler und räumlich unabhängig zu gewähren, agile Organisationsformen und partizipative Entscheidungsmechanismen und Formen der Selbstorganisation anzuwenden - da Mitarbeiter sich auf diese Weise nicht nur an flexibles und mobiles Arbeiten und somit eine selbstständig strukturierte und zielorientierte Arbeitsweise gewöhnen, sondern bei ihnen auch die Ausbildung motivationaler Kompetenzen gefördert wird (Berend und Brohm-Badry 2020).

Als besonders gewinnbringend hat sich im Masterprogramm Digital Marketing auch das Antrainieren von sozialen Kompetenzen im digitalen Bereich erwiesen: die Fähigkeiten, mithilfe digitaler Technologien zu kommunizieren, zusammenzuarbeiten und Daten bzw. Informationen zu teilen sind von größter Relevanz im digitalen Lehrbetrieb - sowohl für Studierende als auch für Lehrende.

Die Wichtigkeit dieser Soft Skills ist für Unternehmen in gleichem Maße gegeben, stellen sie doch sicher, dass die Qualität von Arbeitsabläufen auch im digitalen Bereich aufrechterhalten bleibt, obwohl Teammitglieder bzw. Kollegen sich nicht mehr in Persona absprechen können.

\section{Fünf Erfolgsfaktoren für die digitale Zusammenarbeit in Unternehmen}

Die nahezu problemlose Umstellung von Blended Learning-Format auf die komplett digitale Lehre im Studiengang Digital Marketing lässt sich auf fünf Erfolgsfaktoren zurückführen. Die ersten beiden Faktoren beziehen sich auf das Unternehmen allgemein und die letzten drei Faktoren auf die individuelle digitale Kompetenz der 
Mitarbeiter. Berücksichtigen Unternehmen diese Aspekte, können sie agiler zwischen Präsenzbetrieb und digitaler Zusammenarbeit wechseln.

1. Funktionierende Infrastruktur: Grundlage jeder agilen Strategie ist die vorhandene und funktionierende Infrastruktur. Wissensmanagementsysteme sowie performante Videokonferenzsysteme gehören ebenso zu dieser Infrastruktur wie die Ausstattung mobiler Arbeitsplätze und die Sicherstellung einer leistungsfähigen Internetverbindung im Home-Office.

2. Digitaler Unternehmensalltag: Unternehmen, die bereits im Normalbetrieb digital zusammenarbeiten, können problemlos auf einen digitalen Betrieb umstellen.

3. Didaktik bei digitalen Besprechungen: Die Fähigkeit digitale Besprechungen zu konzipieren und motivierend zu moderieren ist besonders wichtig für das Ergebnis dieser Besprechungen. Hierbei spielt die Ausrichtung des Besprechungskonzepts an der Zielgruppe (internes Meeting, Kundentermin, Abstimmungsrunde) eine ebenso große Rolle, wie die Fähigkeit Besprechungsinhalte mit aktivierenden Methoden anzureichern. Siehe hierzu die beispielhaften Anwendungsmöglichkeiten in Tab. 1.

4. Netiquette: Schon beim Onboarding neuer Mitarbeiter müssen Unternehmen ihren Anspruch an ein angemessenes und achtendes (respektvolles) Benehmen in der technischen (elektronischen) Kommunikation vermitteln. Dazu gehört, dass man bespricht, wann man die Kamera bei virtuellen Besprechungen anstellt und wann nicht oder dass Mikrofone stumm geschalten sind, wenn man nicht spricht. Im Masterprogramm Digital Marketing wurde beispielsweise festgelegt, dass man ein Profilbild im Videokonferenzsystem hinterlegt. So blickt der Moderator nicht auf anonyme „schwarze Kacheln“, wenn die Kameras ausgeschalten sind, sondern zumindest auf ein Profilbild.

Tab. 1 Besprechungsinhalte und aktivierende Methoden

\begin{tabular}{ll}
\hline Besprechungsinhalt & Aktivierende Methoden \\
\hline Information & Foliengestaltung nach dem Prinzip „Bild vor Wort“ \\
& Alle sieben Minuten Einbringen eines aktivierenden Elements \\
Inspiration & Einbinden von Videos, Audiofiles, Bildern, externen Websites \\
& Abfrage von Erwartungen und Wünschen \\
& Einbringen persönlicher Sichtweisen, Anregen zu offenen Diskussionen \\
Teaming & Spielerische Ein- und Ausstiegsrunden (am besten mit aktivierter Webcam) \\
& Bewusste Einrichtung von Zeitslots für, ,Office-Gossip“ \\
& Abfragen von (Vor-) Wissen, Vorerfahrungen oder Meinungen via Abstimmungs- \\
& tools oder Chat \\
Arbeiten in Gruppen/Breakout Sessions \\
Gamification-Elemente wie Quizze, Bewegungsspiele \\
Problemlösung/ & Gemeinsames Brainstorming bzw. Brainwriting \\
Verbesserung & Arbeiten mit digitalen Pinnwänden bzw. Mindmaps \\
& Einsetzen von (anonymen) Q \& A-Tools \\
Bewertung/ & Nutzen von Punkteabfragen, Skalierungsabfragen \\
Commitment/ & Abfragen von Zustimmung oder Ablehnung als Blitzlicht via Status-Icons \\
Entscheidung & Einbinden von Audience Response Systemen wie Pingo oder Kahoot \\
\hline
\end{tabular}


5. Selbstdisziplin: Eine selbständige, eigenverantwortliche und strukturierte Arbeitsweise ist im normalen Betriebsalltag wichtig, jedoch umso wichtiger, wenn dieser Alltag digital stattfindet. Es lohnt sich also, Mitarbeiter auch während des regulären Betriebsablaufs in der Ausbildung dieser Kompetenz zu unterstützen, z.B. durch den Einsatz von New Work-Ansätzen.

Mithilfe dieser Erfolgsfaktoren wird ein Unternehmen also nicht nur in seinen Strukturen digitalisiert und agilisiert, sondern auch hinsichtlich der Kompetenzen seiner Mitarbeiter bereichert. Die Umsetzung der fünf genannten Faktoren birgt damit das Potenzial einer Steigerung der Zufriedenheit der Mitarbeiter und wappnet ein Unternehmen nicht nur gegen Krisenzeiten wie sie die Corona-Pandemie mit sich gebracht hat, sondern stellt eine generelle Investition in die eigene Wettbewerbsfähigkeit dar.

Funding Open Access funding enabled and organized by Projekt DEAL.

Open Access Dieser Artikel wird unter der Creative Commons Namensnennung 4.0 International Lizenz veröffentlicht, welche die Nutzung, Vervielfältigung, Bearbeitung, Verbreitung und Wiedergabe in jeglichem Medium und Format erlaubt, sofern Sie den/die ursprünglichen Autor(en) und die Quelle ordnungsgemäß nennen, einen Link zur Creative Commons Lizenz beifügen und angeben, ob Änderungen vorgenommen wurden.

Die in diesem Artikel enthaltenen Bilder und sonstiges Drittmaterial unterliegen ebenfalls der genannten Creative Commons Lizenz, sofern sich aus der Abbildungslegende nichts anderes ergibt. Sofern das betreffende Material nicht unter der genannten Creative Commons Lizenz steht und die betreffende Handlung nicht nach gesetzlichen Vorschriften erlaubt ist, ist für die oben aufgeführten Weiterverwendungen des Materials die Einwilligung des jeweiligen Rechteinhabers einzuholen.

Weitere Details zur Lizenz entnehmen Sie bitte der Lizenzinformation auf http://creativecommons.org/ licenses/by/4.0/deed.de.

\section{Literatur}

Baltes G, Freyth A (2017) Veränderungsintelligenz Veränderungsintelligenz. Agiler, Innovativer, Unternehmerischer Den Wandel Unserer Zeit Meistern. Springer Gabler, Wiesbaden

Berend B, Brohm-Badry M (2020) New Work: Souveränität im postdigitalen Zeitalter. Springer, Wiesbaden

DeSouza E, Fleming M (2003) A comparison of in-class and Online quizzes on student exam performance. J Comput High Educ 14(2):121-134

Erpenbeck J, Sauter S, Sauter W (2015) E-Learning und Blended Learning. Springer, Wiesbaden

European Center for Digital Competitiveness (2020) Digital Riser Report 2020: Digital etablierte Länder sehen sich mit neuen, dynamischen Wettbewerbern konfrontiert. European Center for Digital Competitiveness, Berlin

Gerner V, Jahn D, Schmidt C (2019) Blended Learning: Die richtige Mischung macht's! Ein praktischer Ideengeber für digital unterstützte Lehr-/Lernkonzepte

Gerstbach I (2020) Die Kunst der Online-Moderation. Tools, Ideen und Tipps für die erfolgreiche Umsetzung. Carl Hanser, München

Harnacke U (2020) Online-Meetings und -Seminare: Effizient und fesselnd gestalten. Haufe-Lexware, Freiburg

it-daily.net (2020) Corona treibt Digitalisierung voran - nicht alle Unternehmen halten mit. https:// www.it-daily.net/it-management/digitalisierung/26196-corona-treibt-digitalisierung-voran-nichtalle-unternehmen-halten-mit. Zugegriffen: 11. Jan. 2021 
Kerres M (2018) Mediendidaktik. Konzeption und Entwicklung digitaler Lernangebote. De Gruyter, Berlin/Boston

Kirchherr J, Klier J, Lehmann-Brauns C, Winde M (2019) Future Skills: Welche Kompetenzen in Deutschland fehlen. https://scholar.google.de/scholar?q=future+skills+welche+kompetenzen+in+deutschland +fehlen\&hl=de\&as_sdt=0\&as_vis=1\&oi=scholart. Zugegriffen: 12. Jan. 2021

Kreulich K, Lichtlein L, Zitzmann C, Bröker T, Schwab R, Zinger B (2020) Hochschullehre in der Post-Corona-Zeit. Studie der Bayerischen Hochschulen für Angewandte Wissenschaften. https://w3-mediapool.hm.edu/mediapool/media/baukasten/img_2/fidl/dokumente_121/ FIDLStudiePostCoronaGesamt.pdf. Zugegriffen: 11. Jan. 2021

Meier S, Lütolf D, Schillerwein S (2015) Herausforderung Intranet: Zwischen Informationsvermittlung, Diskussionskultur und Wissensmanagement. Springer Gabler, Wiesbaden

Neun W (2020) Digitale Transformation und Agilität in der Praxis. Springer Gabler, Wiesbaden

Wiesinger A, Stern S, Daube M, Klier J, Hartmann V (2020) Die Chance für den digitalen Durchbruch. Was Schulen und Politik aus der COVID-19-Krise lernen können. https://www.mckinsey.de/ /media/ mckinsey/locations/europe and middle east/deutschland/publikationen/2020-06-19 digitalisierung in den schulen/202006_die chance fr den digitalen durchbruch.pdf. Zugegriffen: 11. Jan. 2021 